Agricultural Journal 7 (6): 394-400, 2012

ISSN: $1816-9155$

(C) Medwell Journals, 2012

\title{
Construction and Identification of Prokaryotic and Eukaryotic Expression Vectors of Porcine Stratifin
}

\author{
${ }^{1}$ YaLin He, ${ }^{2}$ Mengliang Liu, ${ }^{2}$ XiaoDi Wang, ${ }^{2}$ ShanShan Xu and ${ }^{2}$ Zhiwen Xu \\ ${ }^{1}$ Institute of Animal Nutrition, ${ }^{2}$ Animal Biotechnology Center, \\ Sichuan Agricultural University, 625014 Yaan, China
}

\begin{abstract}
Prokaryotic expression vector of porcine stratifin was constructed to express stratifin in Escherichia $\operatorname{coli}(E$. coli) and eukaryotic expression vector was also constructed. Stratifin cDNA was amplified using the PCR and cloned into pET32a (+) vector to form the recombinant plasmid SFN-pET32a (+); the recombinant plasmid SFN-pET32a (+) was then transformed into prokaryotic expression host $E$. coli Rosetta using lactose induction, the target protein was purified by using his affinity chromatographic separation. The recombinant protein stratifin was identified by SDS-PAGE. Stratifin cDNA was inserted into pcDNA3.1 $(+)$ to form the recombinant plasmid SFN-pcDNA3.1 $(+)$ and then it was identified by restriction enzyme digestion and DNA sequencing. The recombinant plasmid stratifin was successfully constructed, the recombinant stratifin protein was induced and eukaryotic expression vector was constructed. This study successfully realizes the construction of the prokaryotic and eukaryotic expression vectors and it could be used as a foundation for the future research on the function of this protein.
\end{abstract}

Key words: Porcine stratifin, prokaryotic expression, 14-3-3 protein, vector, plasmid, China

\section{INTRODUCTION}

Members of the 14-3-3 protein family form a group of highly conserved $30 \mathrm{kDa}$ acidic proteins expressed in a wide range of organisms and tissues. The five major mammalian brain 14-3-3 isoforms are named $\alpha-\eta$ after their respective elution positions on HPLC (Ichimura et al., 1988; Toker et al., 1992). $\alpha$ and $\delta$ are the phosphoforms of $\beta$ and $\zeta$, respectively (Aitken et al., 1995). Two other isoforms $\tau$ (also known $\theta$ ) and $\delta$ are expressed in $\mathrm{T}$ cells and epithelial cells, respectively, although the former is also widely expressed in other tissues including brain (Boston et al., 1982a, b; Yaffe, 2002). The 14-3-3 is now established as a family of dimeric proteins that can modulate interaction between proteins (including oncogene products of polyoma middle T, Raf-1, AKT and Bcr-Abl). They are involved in cell signalling, regulation of cell cycle progression, intracellular trafficking/targeting, cytoskeletal structure and transcription (Wu et al., 1997; Aitken et al., 1992). In many cases, the interacting proteins show a distinct preference for a particular isoform (s) of 14-3-3 (Daugherty et al., 1996; Ferl, 1996; Liu et al., 1995). A specific repertoire of dimer formation may influence which of the 14-3-3 interacting proteins could be brought together (Xiao et al., 1995). The regulation of interaction usually involves phos-phorylation of the interacting protein and in some cases the phosphorylation of 14-3-3 isoforms themselves may modulateinteraction (Yaffe et al., 1997; Muslin et al., 1996; Aitken et al., 2002).

The name 14-3-3 was given to an abundant mammalian brain protein family due to its particular elution and migration pattern on two-dimensional DEAE-cellulose chromatography and starch gel electrophoresis. The 14-3-3 proteins elute in the 14th fraction of bovine brain homogenate from the researchers homemade DEAEcellulose column and fractions 3.3 in the latter step. Members of the family have been given many other names when they have been rediscovered by other researchers due to their involvement in novel regulatory roles. The first function ascribed to this family of proteins was activation of tyrosine and tryptophan hydroxylases, the rate limiting enzymes involved in catecholamine and serotonin biosynthesis, essential for the synthesis of dopamine and other neurotransmitters (Ichimura et al., 1987).

The 14-3-3 proteins comprise a family of highly conserved acidic protein are widely found in different eukaryotic cells. The 14-3-3 proteins were the first polypeptides shown to have phosphoserine/threonine

Corresponding Author: Zhiwen $\mathrm{Xu}$, Key Laboratory of Animal Disease and Human Health of Sichuan Province, Animal Biotechnology Center, College of Veterinary Medicine, Sichuan Agricultural University, 46\# Xinkang Road, Yucheng District, 625014 Yaan, Sichuan Province, P.R. China 
(pSer/Thr) binding properties which firmly established its importance in cell signalin (Pallas et al., 1994). The 14-3-3 proteins tend proteins processes as metabolism, signal transduction, cell cycle control, cell growth and differentiation, apotosis, protein trafficking, transcription, stress responses and malignant transformation (Kong and Zhang, 2007). Many reports link 14-3-3 to disorders particularly the neurological disorders and cancer. The 14-3-3 test has been used for the diagnosis of prion diseases. The 14-3-3 could be exploited for therapeutic.

Porcine stratifin protein belongs to the 14-3-3 protein, this study mainly focused on the $\delta$ subtypes of 14-3-3 protein aims at constructing the porcine stratifin gene eukaryotic and prokaryotic expression vector for further studies of the protein and the role of foundation.

\section{MATERIALS AND METHODS}

Strains and plasmids: E.coli DH5 $\alpha$, E.coli Rosetta, plasmids pET32a $(+)$ and pcDNA3.1 $(+)$ were preserved at Animal Biotechnology Center, Sichuan Agricultural University (Yaan, China).

Reagents: Trizol was purchased from invitrogen; restriction enzymes EcoR and Hind, pMD19 and PCR marker were purchased from TaKaRa Biotechnology (Dalian) CO., Ltd. Plasmid Mini kit and universal DNA purification kit were purchased from Tiangen Biotech., (Beijing) CO., Ltd.

\section{Prokaryotic expression of porcine stratifin}

PCR primer design: According to GenBank series of mRNA of porcine stratifin, the specific PCR primers of the conserved region was designed the sequence of the upstream primer was P1: 5'-GGAATTCATGGAGAGAG CCAGTGTGATCCAG-3' and that of the downstream primer was P2: 5'-ACAAGCTTTCAGCTCTGGGGCTCC TCAG-3'; restriction sites of EcoR and protective bases were introduced to the $5^{\prime}$ end of upstream primer and Hind and protective bases to the $5^{\prime}$ end of downstream primer. The primer was synthesized by Sangon Biotech (Shanghai) Co., Ltd.

Extraction of total RNA and PT-PCR: Swine blood was collected in aseptic conditions, the total RNA was extracted using one-step Trizol, cDNA was obtained by RT-PCR and the specific PCR primers of the conserved region of porcine stratifin was applied by PCR. The reaction was carried out with the following reaction cycles: 30 consecutive cycles of denaturation at $94^{\circ} \mathrm{C}$ for $30 \mathrm{sec}$, annealing at $55^{\circ} \mathrm{C}$ for $45 \mathrm{sec}$, extension at $72^{\circ} \mathrm{C}$ for
$60 \mathrm{sec}$. About $10 \mu \mathrm{L}$ of PCR products were separated on $1.0 \%$ agarose gel and the result was then obtained with the ultraviolet lamp.

The cloning of PCR products into plasmids pET32a using T-A cloning: PCR products were purified and cloned into plasmids pET32a by T-A cloning. The obtained ligation products were transformed into competent cells $E$. coli $\mathrm{DH} 5 \alpha$. After culture, a single colony was chosen plasmids were extracted which was then digested by EcoR and Hind. The positive recombinants were named PMD19T-SFN and sent to Sangon Biotech (Shanghai) Co., Ltd for sequencing.

Construction of SFN expression vector: PMD19T-SFN and expression plasmid pET32a $(+)$ were digested with EcoR and Hind and after gel recovery, the fragments were used to transformed the competent cells $E$. col $i$ Rosetta.

Expression and identification of SFN fusion proteins: Recombinant strains were selected for monoclonal cultivation by inoculation into $5 \mathrm{~mL} \mathrm{LB} / \mathrm{Amp}$ medium and cultured at $37^{\circ} \mathrm{C}$ for $180 \mathrm{r} \mathrm{min}^{-1}$. After overnight culture, the culture material were inoculated into $100 \mathrm{~mL}$ of LB/Amp medium at $2 \%$ and cultured at $37^{\circ} \mathrm{C}$ until $\mathrm{OD}_{600}=0.5 \sim 0.8$. IPTG was added to final concentration $0.8 \mathrm{mmol} \mathrm{L}^{-1}$; the expression of fusion proteins was induced for additional $3 \mathrm{~h}$ at $37^{\circ} \mathrm{C}$. Culture, without strains, vectors and inducer IPTG was used as control. The bacteria were collected after centrifugation, the supernatant and precipitation were separated and collected, respectively after ultrasonic cytolysis (ice-water bath) in phosphate buffer solution ( $\mathrm{pH} \mathrm{8.0)}$ and centrifugation. The expression of the target protein was identified by SDS-PAGE.

Optimization of expression conditions of fusion proteins: According to the various factor levels which affected the prokaryotic expression of proteins and the expression levels of fusion proteins under different conditions were analyzed in order to discover the optimal expression condition. The conventional molecular biology operations-endonuclease reaction, ligation reaction, preparation of competent cells, plasmid transformation, agarose gel electrophoresis, SDS-PAGE, etc. In above methods used molecular cloning; a laboratory manual as reference.

The construction of eukaryotic expression vector SFN-pcDNA3.1 (+)

PCR primer design: According to GenBank series of mRNA of porcine stratifin, the specific PCR primers of the 
conserved region was designed; the sequence of the upstream primer was P1: 5'-CGGGATCCACCATGGAGA GAGCCAGTGTGATCCAG-3' and that of the downstream primer was P2: 5'-CCG CTCGAGTTATCAGCTCTGGGG CTCCTCAG-3'; restriction sites of BamH and protective bases were introduced to the $5^{\prime}$ end of upstream primer and Xho and protective bases to the $5^{\prime}$ end of downstream primer. The primer was synthesized by Sangon Biotech (Shanghai) Co., Ltd.

PCR reaction: Recombinant plasmid SFN-pET32a (+) were used as template and specific primers were utilized for the amplification. The reaction was achieved with the following reaction cycles: 30 consecutive cycles of denaturation at $94^{\circ} \mathrm{C}$ for $30 \mathrm{sec}$, annealing at $57^{\circ} \mathrm{C}$ for $45 \mathrm{sec}$, extension at $72^{\circ} \mathrm{C}$ for $60 \mathrm{sec}$. A total of $10 \mu \mathrm{L}$ of PCR products were separated on $1.0 \%$ agarose gel and the result was then obtained with the ultraviolet lamp.

Construction of eukaryotic plasmid SFN-pcDNA3.1 (+): PCR products were purified; pcDNA3.1 $(+)$ and PCR products were digested with BamH and Xho. The agarose gel electrophoresis was conducted and after gel recovery, the fragments were used to transform the competent cells $E$. coli $\mathrm{DH} 5 \alpha$. The plasmids were extracted and sent to Sangon Biotech (Shanghai) Co., Ltd for sequencing.

\section{RESULTS AND DISCUSSION}

\section{Prokaryotic expression of stratifin}

Ex vivo expression of stratifin: Through the detection of $1.0 \%$ agarose gel electrophoresis, the amplified DNA products were about $750 \mathrm{bp}$ and the sizes of the amplified products were in accordance with those of the desired products (Fig. 1).

Identification of recombinant plasmid SFN-pET32a (+): The extraction was digested with EcoR and Hind identified by $1.5 \%$ agarose gel electrophoresis and two DNA fragments of about $5.9 \mathrm{~kb}$ and $750 \mathrm{bp}$ were obtained the sizes of which were in accordance with desired products (Fig. 2). Compared with the series of stratifin provided by PubMed, the series of coding gene, after the sequencing, equated with the provided series in other words, the recombinant plasmids were successfully constructed and were named SFN-pET32a $(+)$.

The expression of proteins and optimization of expression conditions: The recombinant strains SFN-pET32a (+) Rostta with recombinant plasmids were induced and

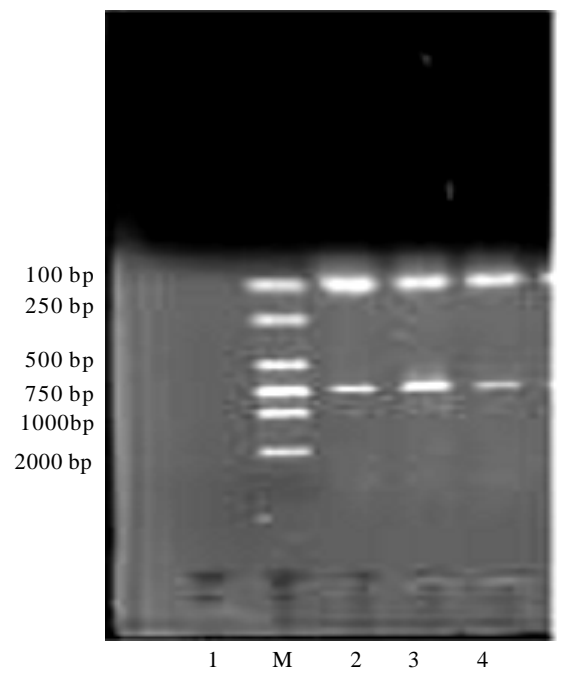

Fig. 1: 1: Negative control; M: DL Marker 2003; 2-4: PCR amplified products

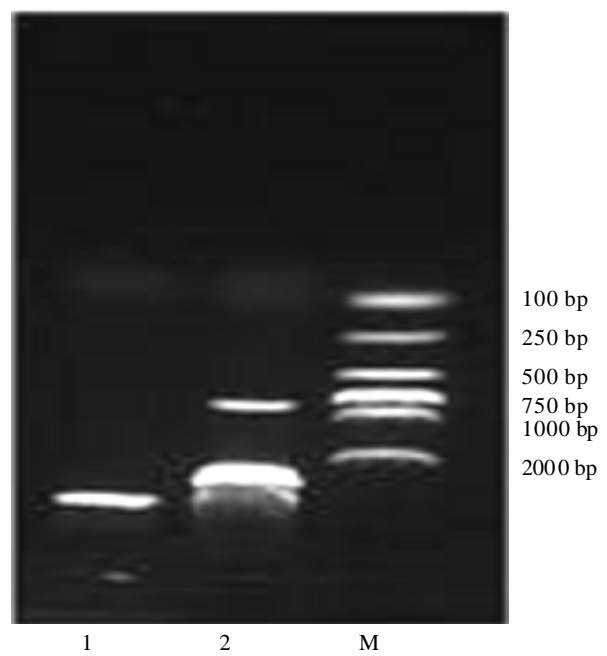

Fig. 2: 1: SFN-pET32a (+); 2: digested by EcoR and Hind; 3: DL Maker 2000

broken up and the supernatant and precipitation were separated and collected, respectively. The precipitation was dissolved by urea and SDS-PAGE was performed (Fig. $3 \mathrm{a}$-e). A protein with an approximate size of $45 \mathrm{kD}$, as indicated by the arrow in the Fig. 3, was expressed by the recombinant strains the expression was mainly restricted in the supernatant. The study optimized the condition related to induction period, IPTG concentration and cell concentration.

Identification of recombinant proteins: The expressed proteins were purified with his affinity chromatography 

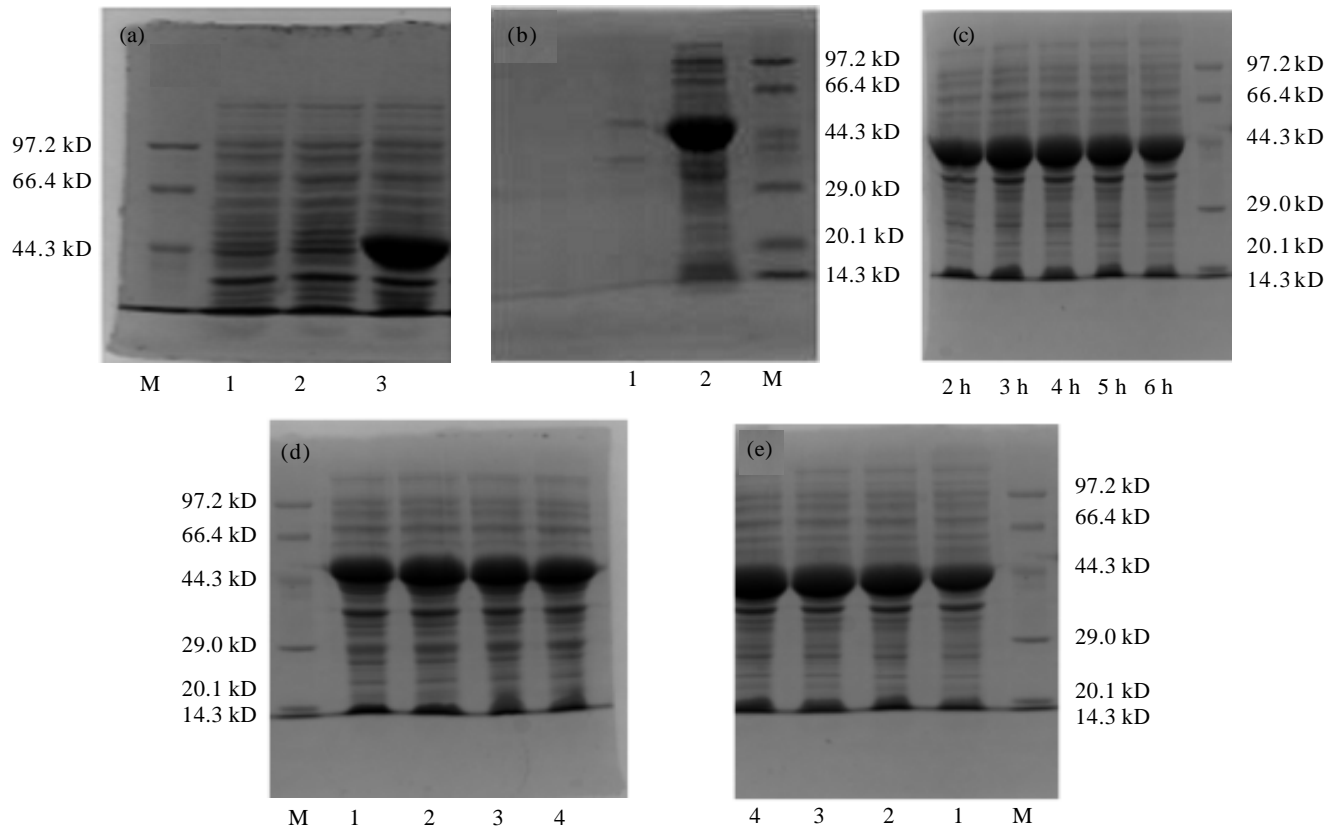

Fig. 3: The precipitation dissolved by urea and SDS-PAGE performed; a) M: low molecular weight protein marker; 1: On-load PET32; 2: Before induction; 3-5 h after 1 mmol L ${ }^{-1}$ PTG induction; b) 1: Precipitation; 2: Supernatant; M: Low molecular weight protein marker, c) Optimization of induction period: 2-6 h, respectively; d) Optimization of IPTG concentration 1: $1 \mathrm{mmol}$; 2: $0.8 \mathrm{mmol}$; $3: 0.6 \mathrm{mmol}$; $4: 0.4 \mathrm{mmol}$; e) Optimization of cell concentration 1: $\mathrm{OD}_{600}=0.5 ; 2: \mathrm{OD}_{600}=0.5 ; 3: \mathrm{OD}_{600}=0.7 ; 4: \mathrm{OD}_{600}=0.8$

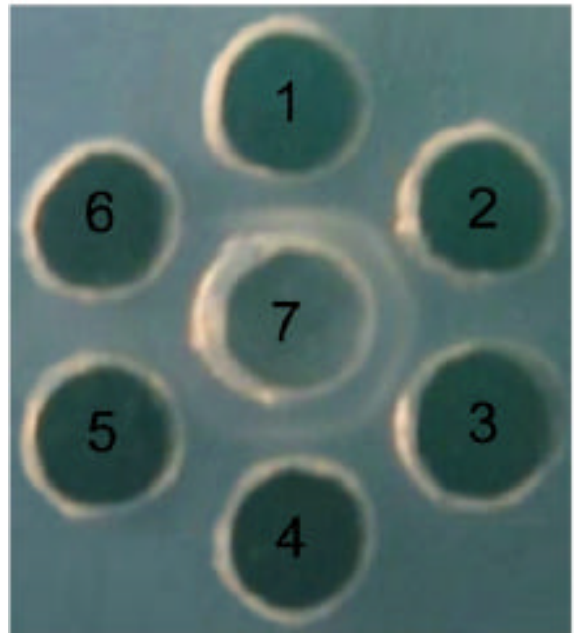

Fig. 4: 1-6: Standard serum concentration was diluted by serial dilution; 7: Recombinant proteins

column and agarose gel electrophoresis with purchased standard serum was performed to identify the expressed proteins. The white immunoprecipitation zone indicted that the recombinant proteins could interact with the standard serum and had certain immunogenicity. The results were shown in Fig. 4.

\section{Construction of eukaryotic expression vector}

Ex vivo expression of stratifin: Through the detection of $1.0 \%$ agarose gel electrophoresis, the amplified DNA products were about $750 \mathrm{bp}$ and the sizes of the amplified products were in accordance with those of the desired products (Fig. 5).

Identification of recombinant plasmid SFN-pcDNA3.1 $(+)$ : The extraction was digested with BamH and Xho, identified by $1.2 \%$ agarose gel electrophoresis and two DNA fragments of about $5.4 \mathrm{~kb}$ and $750 \mathrm{bp}$ were obtained, the sizes of which were in accordance with desired products (Fig. 6). Compared with the series of stratifin provided by PubMed, the series of coding gene, after the sequencing, equated with the provided series in other words, the recombinant plasmids were successfully constructed and were named SFN-pcDNA3.1 (+).

The 14-3-3 proteins are high conservative proteins that can be found in all eukaryotic cells in most living, they belong to a class of regulatory proteins encoded by a super-family gene. The proteins participate in almost all physiological processes; researchers have discovered a wide variety of 14-3-3 proteins in various tissue cell. As the first signaling molecules binding to phosphoserine/ threonine, the 14-3-3 proteins play vital role in signal 
Agric. J., 7 (6): 394-400, 2012

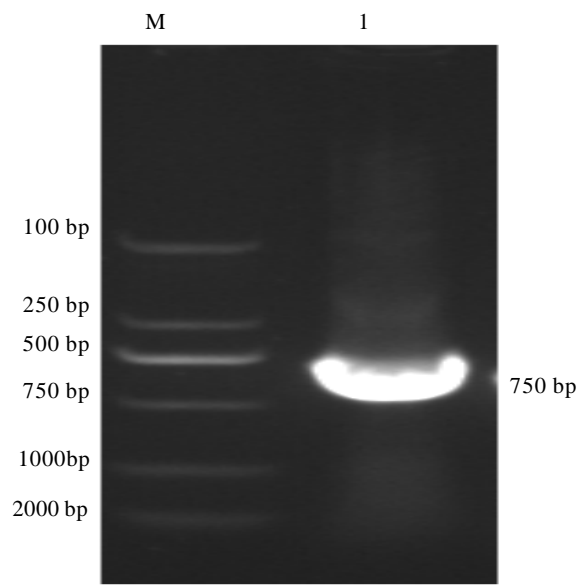

Fig. 5: M: DL Maker 2000; 1: PCR amplified products

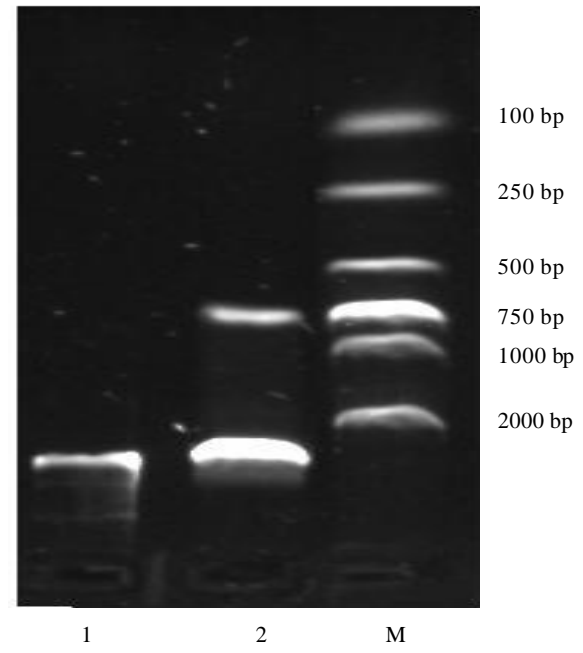

Fig. 6: 1: SFN-pcDNA3.1 (+); 2: digested with BamH and Xho; M: DL Maker 2000

transduction in cells, especially in its participation in directly regulating the activity of protein kinases and phospholipases and are known as the bridge protein. Many studies show that the regulatory mechanisms of the 14-3-3 proteins can lead to the occurrence of certain diseases (Selleck and Tan, 2008).

The 14-3-3 proteins belong to a protein family that is widely distributed in eukaryotic animals and serves several biological functions in organisms. The 14-3-3 proteins are able to interact with certain regulatory factors in various pathways and links and inhibit apoptosis; the proteins participate in the regulation of apoptosis through the following modes of action; the proteins control the sub-cellular location of target proteins (Bad, Bax, Fkhrl 1, etc.) and serve as adaptor proteins (A20, c2Raf21, etc.) promoting the interaction between proteins and regulating the activity of key enzymes (ASK1, etc.); however, the specific mechanism of the proteins still needs further study. Till now, many diseases will show the process of apoptosis further researches are needed to determine whether these proteins can be used in the ancillary treatment.

Recent studies showed that the infection with PCV2 in swine would lead to an up-regulation of the 14-3-3 proteins in host cells. The up-regulation might be a strategy PCV2 with the help of its antiapoptotic effect utilizes to escape from the immune surveillance of the host cells to complete its proliferation or it might be that the existence of the interaction between the 14-3-3 proteins and MEKK proteins may help to regulate MAPK signal pathway. The integration effect of the 14-3-3 proteins might be widely seen not only in the crosswalk among various signal pathways but also in the whole complex signaling transduction networks. With the help of the protein technique and the existing genomic data, people will discover more proteins that are able to interact with the 14-3-3 proteins. In the future, signal regulatory networks mediate by the 14-3-3 proteins will be further expanding.

In the prokaryotic expression system, not only an appropriate expression vector is needed but also a suitable host strain in order to obtain the effective expressive effects which is another essential link to a successful experiment. When expressed in the host strains excess in endogenous protease will serve as the potential threat to expressed products as it may be degraded. In this research, the host strains Rosetta (DE3) were derived from the expressing strains BL21 which were ompT and lon protease deficient and in the meantime the strains also have $T 7$ RNA polymerase gene. Most importantly, these strains supply tRNA for AUA, AGA, $\mathrm{AGG}, \mathrm{CCC}, \mathrm{GGA}$ and CUA codons on compatible chloramphenicol-resistant plasmid thus, Rosetta (DE3) strains become the universal translation which is otherwise limited by the codon usage of $E$. coli. As a result, even if carrying $E$. coli rare codon, eukaryotic proteins can still be efficiently expressed in Rosetta (DE3).

When the expressing strains were induced by certain concentration of IPTG the exogenous genes could be expressed. However, IPTG is a kind of alien materials that can be toxic to the expressing strains in high concentration and will affect the expression of the exogenous proteins. As a result, the determination of the optimal concentration of IPTG has very vital practical meanings. Through analysis, the optimal concentration of IPTG in this study is $0.8 \mathrm{mmol} \mathrm{L}^{-1}$. In prokaryotic 
expression, the growth period of cells will intensely affect the expression of exogenous proteins and excessive growth will aggravate stress on the system, leading to the formation of insoluble inclusion bodies. In addition, after long period of expression, metabolic products of the cells are seriously accumulated which adds to difficulty in purification. However, the short expression period will otherwise disable the full expression of the recombinant proteins and the yield of proteins can be low. The long expression period will increase the amount of the metabolic products which is not conductive to the yield and purification of the expressed proteins. In this research, after obtaining the optimal concentration of IPTG, the optimal induction time is finally determined as $3 \mathrm{~h}$. The most important factor that influences the effective expression of exogenous genes in E. coli might be the growth temperature under high temperature, the expressed products are usually inclusion body proteins. Consequently, if the expression of fusion proteins is required lower temperature is recommended. On the basis of the optimal concentration of IPTG and induction time, the optimal expression temperature is determined as $37^{\circ} \mathrm{C}$.

\section{CONCLUSION}

In this study, stratifin genes were cloned from the swine blood and it showed from the results of the sequencing that the homology between stratifin $D Q$ 445254.1 and cloned genes was $99 \%$ which showed that the protein genes were conserved to some extent. In the meantime, the prokaryotic expression of the proteins was successfully achieved which could serve as the foundation for the future researches on their effects in diseases and on whether they could be applied in disease treatment.

\section{ACKNOWLEDGEMENTS}

This research was supported by Program of Changjiang Scholars and innovative Research Team in University (IRT0848), YaLin $\mathrm{He}$ and MengLiang Liu contributed to the research, XiaoDi Wang helped the researchers perform thesections for this research, Shanshan Xu helped read the changes caused by thevirus studied.

\section{REFERENCES}

Aitken, A., D.B. Collinge, B.P. van Heusden, T. Isobe, P.H. Roseboom, G. Rosenfeld and J. Soll, 1992. 14-3-3proteins: A highly conserved, widespread family of eukary-otic proteins. Trends Biochem. Sci., 17: 498-501.
Aitken, A., S. Howell, D. Jones, J. Madrazo and Y. Patel, 1995. 14-3-3a and a are the phosphorylated forms of Raf-activating 14-3-3â and æ in vivo stoichiometric phosphorylation in brain at a Ser-Pro-Glu-Lys MOTIF. J. Biol. Chem., 270: 5706-5709.

Aitken, A., H. Baxter, T. Dubois, S. Clokie, K. Mitchell, A. Peden and E. Zemlickova, 2002. Specificity of 14-3-3 isoform dimer interactions and phosphorylation. Biochem. Soc. Trans., 30: 351-360.

Boston, P.F., P. Jackson, P.A.M. Kynoch and R.J. Thompson, 1982a. Purification, properties and immunohistochemical localization of human brain 14-3-3 protein. J. Neurochem., 38: 1466-1 474.

Boston, P.F., P. Jackson and R.J. Thompson, 1982b. Human 14-3-3 protein: Radioimmunoassay, tissue distribution and cerebrospi-nal fluid levels in patients with neurological disorders. J. Neurochem., 38: 1475-1482.

Daugherty, C.J., M.F. Rooney, P.W. Miller and R.J. Ferl, 1996. Molecular organization and tissue-specific expression of an Arabidopsis 14-3-3 gene. Plant Cell, 8: 1239-1248.

Ferl, R.J., 1996. 14-3-3 proteins and signal transduction. Ann. Rev. Plant Physiol. Plant Mol. Biol., 47: 49-73.

Ichimura, T., T. Isobe, T. Okuyama, T. Yamauchi and H. Fujisawa, 1987. Brain 14-3-3 protein is an activator protein that activtes tryptophan 5-monooxygenase and tryptophan 3-monooxygenase in the presence of $\mathrm{Ca} 2+$ calmodulin-dependent protein kinase II. FEBS Lett., 219: 79-82.

Ichimura, T., T. Isobe, T. Okuyama, N. Takahashi, K. Araki, R. Kuwano and Y. Takahashi, 1988. Molecular cloning of cDNA coding for brain-specific 14-3-3 protein a protrin kinase-dependent activator of tyrosine and tryptophan hydroxylases. Proc. Natl. Acad. Sci. USA., 85: 7048-7088.

Kong, L.Y. and Y.Z. Zhang, 2007. The basics of 14-3-3 protein family and research progress on therapeutic applications of 14-3-3 protein. Sheng Wu Gong Cheng Xue Bao., 23: 781-788.

Liu, D., J. Bienkowska, C. Petosa, R.J. Collier, H. Fu and R. Liddington, 1995. Crystal structure of the zeta isoform of the 14-3-3 protein. Nature, 376: 191-194.

Muslin, A.J., J.W. Tanner, P.M. Allen and A.S. Shaw, 1996. Interaction of 14-3-3 with signaling proteins is mediated by the recognition of phosphoserine. Cell, 84: 889-897.

Pallas, D.C., H. Fu, L.C. Haehnel, W. Weller, R.J. Collier and T.M. Roberts, 1994. Association of polyomavirus middle tumor antigen with 14-3-3 proteins. Science, 265: 535-537. 
Selleck, W. and S. Tan, 2008. Reeombinant protein complex express in E. coli. Curr. Protoc. Protein Sci., 21: 324-331.

Toker, A., L.A. Sellers, B. Amess, Y. Patel, A. Harris and A. Aitken, 1992. Multiple isoforms of a protein kinase Cinhibitor(14-3-3/KCIP) from sheep brain Amino acid sequence of phosporylated forms. Eur. J. Biochem., 206: 453-456.

Wu, K., M.F Rooney and R.J. Ferl, 1997. The Arabidopsis 14-3-3 multigene family. Plant Physiol., 114: 1421-1431.
Xiao, B., S.J. Smerdon, D.H. Jones, G.G. Dodson, Y. Soneji, A. Aitken and S.J. Gamblin, 1995. Structure of a 14-3-3 protein and implications for coordination of multiple signalling pathways. Nature, 376: 188-191.

Yaffe, M.B., K. Rittinger, S. Volinia, P.R. Caron and A. Aitken et al., 1997. The structural basis for 14-3-3 phosphopeptide binding specificity. Cell, 91: 961-971.

Yaffe, M.B., 2002. How do 14-3-3 proteins work?Gatekeeper phos-phorylation and the molecular anvil hypothesis. FEBS Lett., 513: 53-57. 